\title{
IMPACTO DAS MUDANÇAS CLIMÁTICAS NO AMBIENTE TÉRMICO INTERNO DE HABITAÇÃO UNIFAMILIAR EM CUIABÁ-MT
}

\author{
CLIMATE CHANGE IMPACT ON THE INDOOR THERMAL \\ ENVIRONMENT OF UNIFAMILIARY HOUSING IN CUIABÁ-MT
}

\author{
Emeli Lalesca Aparecida da Guarda ${ }^{1}$ \\ Universidade Federal de Santa Catarina, Florianópolis, SC, Brasil, emeliguarda@gmail.com \\ Luciane Cleonice Durante ${ }^{2}$ \\ Universidade Federal do Mato Grosso, Cuiabá, MT, Brasil, luciane.durante@hotmail.com \\ Ivan Julio Apolonio Callejas ${ }^{3}$ \\ Universidade Federal do Mato Grosso, Cuiabá, MT, Brasil, ivancallejas1973@gmail.com
}

\begin{abstract}
Resumo
Objetiva-se nessa pesquisa avaliar os impactos das mudanças climáticas no desempenho termoenergético de habitação de interesse social localizada na cidade de Cuiabá-MT, considerando propostas de adequação de sua envoltória tanto no Cenário Base (1961-1990) quanto nos cenários de prospecção de aquecimento global - 2020 (2011-2040), 2050 (2041-2070) e 2080 (2071-2100). As prospecções foram feitas por meio de simulação computacional, utilizando o software EnergyPlus, seguindo as etapas metodológicas: i) preparação dos arquivos climáticos futuros; ii) definição da tipologia construtiva de habitação de interesse social denominada "HISp"; iii) implementação de adequações na envoltória para enquadramento aos melhores níveis das normativas e regulamentos brasileiros de desempenho termoenergético no Cenário Base, obtendo-se a tipologia denominada "HISa"; iii) simulação computacional da temperatura e umidade do ar externo e no interior dos ambientes de permanência prolongada da habitação; e iv) análise comparativa das intervenções. Os impactos quantificados apontam para uma variação da temperatura externa e umidade relativa do ar média anual de $21,5 \%\left(+5,75^{\circ} \mathrm{C}\right)$ e $22 \%(-15,4 \%)$, respectivamente, até o Cenário 2080 , se comparadas ao Cenário Base. As envoltórias das habitações HISa e HISp classificaram-se como "A" e " $D$ " no Cenário Base e ambas como "E" no Cenário 2080. O consumo relativo de energia para condicionamento térmico artificial provisionado para HISp e da HISa poderá se elevar em $83,75 \%$ e 99,63\%, respectivamente, em 2080, se comparado ao Cenário Base. Prevalecendo as condições climáticas provisionadas, estas poderão ser impeditivas à manutenção da classificação da eficiência energética da envoltória como " $A$ ", desencadeando elevado consumo de energia para resfriamento.
\end{abstract}

Palavras-chave: Resiliência térmica. Envoltória. Sustentabilidade ambiental. Aquecimento global.

\begin{abstract}
The objective of this research is to evaluate the impacts of climate change on the thermal and energectic performance of social housing located in the city of Cuiabá-MT, considering proposals to adapt its envelope both in the Base Scenario (1961-1990) and in the prospecting scenarios of heating global - 2020 (2011-2040), 2050 (2041-2070) and 2080 (2071-2100). Prospecting was done through computer simulation, using the EnergyPlus software, following the methodological steps: preparation of future climate files; definition of the type of constructive housing of social interest called "HISp"; implementation of adjustments in the envelope to fit the best levels of Brazilian norms and regulations for thermal and energectic performance in the Base Scenario, obtaining the typology called "HISa"; computational simulation of the temperature and humidity of the outside air and inside the environments of prolonged residence; and comparative analysis of interventions. The quantified impacts point to an average annual temperature variation and relative humidity of the air of $21.5 \%\left(+5.75^{\circ} \mathrm{C}\right)$ and $22 \%(-15.4 \%)$, respectively, up to Scenario 2080 , if compared to the Base Scenario. The envelopes of the HISa and HISp dwellings were classified as " $A$ " and " $D$ " in the Base Scenario and both as " $E$ " in the 2080 Scenario. The relative energy consumption for artificial thermal conditioning provided for HISp and HISa may rise by $83.75 \%$ and $99.63 \%$, respectively, in 2080 compared to the Base Scenario. If the prevailing climatic conditions prevail, they may impede maintaining the envelope's energy efficiency rating as "A," triggering high energy consumption for cooling.
\end{abstract}

Keywords: Thermal resilience. Building envelope. Environmental sustainability. Global warming.

How to cite this article:

GUARDA, E. L. A. da; DURANTE, L. C.; CALLEJAS, I. J. A. Impacto das mudanças climáticas no ambiente térmico interno de habitação unifamiliar em Cuiabá-MT. PARC Pesquisa em Arquitetura e Construção, Campinas, SP, v. 11, p. e020031, 2020. DOI: http://dx.doi.org/10.20396/parc.v11i0.8657188 


\section{Introdução}

À medida que pesquisas científicas evidenciam um aumento progressivo da temperatura terrestre, as mudanças climáticas são reconhecidas como um desafiochave para o século XXI em todos os setores produtivos da sociedade, inclusive no âmbito da indústria da construção civil, que atua em todo o ciclo de vida do ambiente construído - edificações e cidades.

O Quarto Relatório de Avaliação das Mudanças Climáticas (AR4), intitulado "Fourth Assessment Report: Climate Change 2007" (IPCC, 2012) propôs modelos para avaliar os impactos do aquecimento global, a partir de dados históricos, demográficos, sociais, econômicos e tecnológicos. Esses modelos são denominados cenários A1, A2, B1 e B2, onde 'A' e 'B' significam baixo e alto comprometimento com o desenvolvimento sustentável, e '1' e ' 2 ', a integração ou fragmentação regional, respectivamente. Os cenários de classificação 'A' são considerados 'pessimistas' e os cenários de classificação 'B' são considerados 'otimistas'. Essa publicação destaca que a maior parte dos eventos de aumento da temperatura observados nos últimos 50 anos foi provocada pelas ações antrópicas e alerta para o aumento médio das temperaturas globais de $1,1^{\circ} \mathrm{C}$ a $5,4^{\circ} \mathrm{C}$, podendo atingir $6,4^{\circ} \mathrm{C}$ até o ano de 2100 , se a população e a economia continuarem em acelerado crescimento, mantendo o consumo intenso de combustíveis fósseis (IPCC, 2007).

Considerando que a vida útil dos edifícios é de 50 a 75 anos (ABNT, 2013), infere-se que o desempenho das edificações atuais será afetado pelas mudanças climáticas previstas para as próximas décadas. Portanto, faz-se necessário o estudo do comportamento das edificações nessas condições ambientais futuras, mediante alterações na temperatura do ar externo e nas demais variáveis climatológicas.

Sabe-se que o desempenho térmico e energético dos edifícios é dependente do clima ao qual estão expostos (OLGYAY, 1973; MACIEL; FORD; LAMBERTS, 2007; DE WILDE; COLEY, 2012) e, portanto, novas condições climáticas irão influenciar e impor novos impactos aos edifícios e aos usuários (ALVES, 2014; GUARDA et al., 2019). Nico-Rodrigues (2015) afirma que adotar diretrizes que consideram as relações entre o clima e os seres humanos é um importante instrumento para definição de habitações adequadas às condições de conforto térmico. Assim, pesquisas que abordam esse tema na escala do ambiente construído têm atuado no prognóstico das temperaturas urbanas por meio de modelos matemáticos (DU; EDGE; UNDERWOOD, 2011; KERSHAW; EAMES; COLEY, 2011; ROBERT; KUMMERT, 2012) e, mais recentemente, têm focado nas simulações de desempenho termoenergético de edifícios mediante os efeitos do aquecimento global, estabelecendo prognósticos das condições de conforto e segurança dos ambientes internos, consumo de energia elétrica (WANG; LIU; BROWN, 2017; SONG;YE, 2017), além de intensificar estudos sobre estratégias de projeto passivas para proporcionar condições mais adequadas de habitabilidade como forma de mitigação (CASAGRANDE; ALVAREZ, 2013; TRIANA; LAMBERTS; SASSI, 2016; INVIDIATA ; GHISI, 2016; FAHMY et al., 2017; SABUNAS ; KANAPICKAS, 2017).

Assim sendo, o objetivo deste estudo é analisar o impacto das mudanças climáticas no desempenho termoenergético de uma habitação unifamiliar de interesse social localizada na cidade de Cuiabá-MT, considerando propostas de adequação de sua envoltória aos Cenários Base (1961-1990) e Futuros - 2020 (2011-2040), 2050 (2041-2070) e 2080 (2071-2100), conforme previsão de emissões A2 do Quarto Relatório do IPCC (AR4). 


\section{Referenciais teórico-metodológicos}

As normativas e regulamentos adotados como referenciais teóricos neste estudo apresentam diretrizes construtivas e estratégias passivas de projeto para adequação das edificações ao clima dos locais em que se inserem: a NBR 15.220 (ABNT, 2005), a NBR 15.575 (ABNT, 2013) e o Regulamento Técnico de Qualidade para o Nível de Eficiência Energética de Edificações Residenciais (RTQ-R) (INMETRO, 2012). Todas fornecem recomendações mínimas de transmitância térmica, capacidade térmica e absortância para alcançar condições mínimas de desempenho das edificações habitacionais.

A NBR 15.220 (ABNT, 2005) classifica o país em oito Zonas Bioclimáticas (Z1 a Z8), compreendidas como regiões do território que possuem requisitos similares a serem atendidos pelas edificações nelas localizadas, para proporcionar a adequação ao clima (ABNT, 2005). Desta forma, o estado de Mato Grosso, pela sua diversidade de bioma e localização geográfica, compreende cinco zonas bioclimáticas, a saber: 3, 5, 6, 7 e 8. O município de Cuiabá-MT está inserido na Z7 e os valores das propriedades termo físicas para as vedações verticais e horizontais das normatizações e regulamentos estão descritas na Tabela 1.

\begin{tabular}{|c|c|c|c|c|}
\hline Envoltória & Referência & Absortância solar & $\begin{array}{c}U \\
\left(W / m^{2} K\right)\end{array}$ & $\mathrm{C}\left(\mathrm{kJ} / \mathrm{m}^{2} \mathrm{~K}\right)$ \\
\hline \multirow{7}{*}{ 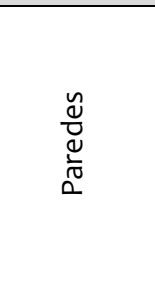 } & \multirow{3}{*}{ NBR 15.220} & Para inércia leve FSo* $\leq 5,0$ & & Para inércia leve $\phi^{* *} \leq 4,3$ \\
\hline & & Para inércia média FSo $\leq 4,0$ & $\leq 2,20$ & Para inércia média $\phi \leq 4,3$ \\
\hline & & Para inércia pesada FSo $\leq 3,5$ & & Para inércia pesada $\phi \geq 6,5$ \\
\hline & \multirow{2}{*}{ NBR 15.575} & $\leq 0,6$ & $\leq 3,70$ & \multirow{4}{*}{$>130$} \\
\hline & & $>0,6$ & $\leq 2,50$ & \\
\hline & \multirow{2}{*}{ RTQ-R } & $\leq 0,6$ & $\leq 3,70$ & \\
\hline & & $>0,6$ & $\leq 2,50$ & \\
\hline \multirow{7}{*}{ 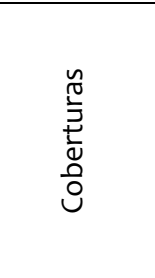 } & \multirow{3}{*}{ NBR 15.220} & Para inércia leve FSo* $\leq 6,5$ & & Para inércia leve $\phi^{* *} \leq 3,3$ \\
\hline & & Para inércia média FSo $\leq 6,5$ & $\leq 2,20$ & Para inércia média $\phi \leq 43,3$ \\
\hline & & Para inércia pesada FSo $\leq 6,5$ & & Para inércia pesada $\phi \geq 6,5$ \\
\hline & \multirow{2}{*}{ NBR 15.575} & $\leq 0,4$ & $\leq 2,30$ & \multirow{4}{*}{ Não especificado } \\
\hline & & $>0,4$ & $\leq 1,50$ & \\
\hline & \multirow{2}{*}{ RTQ-R } & $\leq 0,4$ & $\leq 2,30$ & \\
\hline & & $>0,4$ & $\leq 1,50$ & \\
\hline
\end{tabular}

*FSo: Fator Solar; ** $\varphi$ : Atraso térmico. Fonte: adaptado de NBR 15.220 (ABNT, 2005), NBR 15.575 (ABNT, 2013) e RTQ-R (INMETRO, 2012).

O RTQ-R tem como objetivo avaliar o nível de eficiência energética das edificações residenciais e apresenta método de simulação que se baseia no cálculo do indicador de Graus-Hora de Resfriamento e/ou Aquecimento para avaliar o desempenho do edifício em duas condições de operação: Naturalmente Ventilada (NV) ou Condicionada Artificialmente (HVAC).

Calcula-se o indicador para cada ambiente de permanência prolongada, a partir da temperatura operativa horária $\left(\mathrm{To}, \mathrm{em}{ }^{\circ} \mathrm{C}\right)$, a temperatura média do $\operatorname{ar}\left(\mathrm{Ta}\right.$, em ${ }^{\circ} \mathrm{C}$ ) e a temperatura radiante média do ambiente $\left(\mathrm{Tr}, \mathrm{em}^{\circ} \mathrm{C}\right)$ (Equação 1$)$.

$$
T o=A \times T a+(1-A) \times T r
$$

Equação 1

O software EnergyPlus, fornece a temperatura operativa de cada ambiente em ${ }^{\circ} \mathrm{C}$, portanto, para obter o indicador o RTQ-R indica em realizar o somatório da diferença entre a temperatura operativa horária (To) e a temperatura base $\left(26^{\circ} \mathrm{C}\right)$ (Equação 2 ). 


$$
G H r=\sum\left(T o-26^{\circ} \mathrm{C}\right)
$$

Equação 2

A partir do indicador de GHR, é possível obter o Equivalente Numérico da Envoltória do Ambiente (EqNumEnvAmb) em função do Equivalente Numérico da Envoltória do Ambiente para Resfriamento (EqNumEnvAmbResfriamento) e do Equivalente Numérico da Envoltória do Ambiente para Aquecimento (EqNumEnvAmbAquecimento). Para a ZB7, o RTQ-R indica utilizar o valor do EqNumEnvAmb igual ao EqNumEnvAmbResfriamento, para obter a classificação de eficiência energética. O Equivalente Numérico da Envoltória (EqNumEnv) é obtido por meio da ponderação do EqNumEnvAmbResfriamento pela área útil de cada ambiente de permanência prolongada, variando do nível $A$ (mais eficiente) ao $E$ (menos eficiente) (Quadro 2) (INMETRO, 2012).

Quadro 2 - Equivalente numérico da envoltória do ambiente (NV) para a Zona Bioclimática 7. Fonte: INMENTRO (2012)
\begin{tabular}{|c|c|c|}
\hline Eficiência & $\begin{array}{c}\text { Equivalente Numérico da Envoltória do } \\
\text { Ambiente (EqNumEnvAmb) }\end{array}$ & Condição \\
\hline A & 5 & GHR $\leq 12.566$ \\
\hline B & 4 & $12.566 \leq \mathrm{GHR} \leq 18.622$ \\
\hline C & 3 & $18.622 \leq \mathrm{GHR} \leq 24.679$ \\
\hline D & 2 & $24.679 \leq \mathrm{GHR} \leq 30.735$ \\
\hline E & 1 & $\mathrm{GHR}>30.735$ \\
\hline
\end{tabular}

O RTQ-R define Consumo Relativo (CR) como o consumo anual de energia (em KWh/m²) necessário para refrigeração do ambiente durante o período das 21 às $8 \mathrm{~h}$, ao longo de todos os dias do ano, com manutenção da temperatura em $24^{\circ} \mathrm{C}$ (INMETRO, 2012). A metodologia compara o consumo relativo para refrigeração dos ambientes de permanência prolongada das habitações com o nível de eficiência da envoltória quando a edificação é condicionada artificialmente, sendo o mesmo de caráter apenas informativo e utilizado para obtenção de bonificação para condicionamento de ar.

$O$ consumo relativo estimado de energia elétrica anual e mensal dos ambientes condicionados artificialmente nos padrões estabelecidos pelo RTQ-R (INMETRO, 2012) foram obtidos por meio de simulações computacionais na categoria condicionada artificialmente (HVAC), para o ano todo (8.760 horas) considerando pessoas, equipamentos e iluminação, conforme padrões do RTQ-R. Inicialmente analisou-se o consumo relativo total, somando o consumo dos equipamentos, iluminação e ar condicionado. Em seguida, analisou-se o sistema de HVAC, subtraindo os consumos referentes à iluminação e equipamentos do consumo estimado total, com a finalidade de verificação da demanda energética exclusiva de condicionamento artificial para os cenários climáticos futuros, na condição atual e sob a influência do aquecimento global.

\section{Materiais e métodos}

Essa pesquisa caracteriza-se como de natureza quantitativa e desenvolve-se como uma abordagem por cenários. De acordo com Serra (2006), na abordagem por cenários, se constrói um cenário atual, após levantamento de dados primários e secundários e cenários hipotéticos, que é o estado que se deseja atingir em determinado tempo.

Contextualização climática do estado de Mato Grosso e da cidade de Cuiabá-MT

Para atender aos objetivos da pesquisa, buscou-se compreender o clima da região de estudo, por meio de referências históricas do clima do estado de Mato Grosso e da cidade de Cuiabá-MT. 
Mato Grosso tem cerca de 90\% do seu território inserido no clima do tipo Aw (tropical com chuvas de verão) e cerca de 10\%, no extremo norte, em clima do tipo Am (tropical com clima de monção com precipitação total anual média maior que $1500 \mathrm{~mm}$ e precipitação do mês mais seco menor que 60mm) (PEEL; FINLAYSON; MCMAHON, 2007). Duarte (2000) apresenta que o clima quente é marcante na região de Mato Grosso, apresentando temperaturas diárias altas com frequência, podendo ocorrer máximas superiores a $40^{\circ} \mathrm{C}$ nos meses de setembro e outubro. A autora destaca que nenhum mês apresenta temperatura média inferior a $20^{\circ} \mathrm{C}$ e as temperaturas médias anuais em geral, são elevadas.

A cidade de Cuiabá localiza-se no Centro Sul do estado, localizada em uma província geomorfológica denominada Baixada Cuiabana, na Latitude $15^{\circ} 36^{\prime} 56^{\prime \prime}$ S e Longitude 56 06'01"W. Conforme Callejas et al. (2019), o município situa-se em três importantes ecossistemas brasileiros, o Pantanal ao Sul, a Floresta Amazônica ao Norte e o Cerrado em seu entorno. O clima da cidade é tratado por Durante (2012) que, elaborou estudo comparativo de dados históricos de Campelo Júnior, Priante Filho e Caseiro (1992) referentes ao período de 1970 a 1991 e do Instituto Nacional de Meteorologia (INMET) no período de 2000 a 2010 e observou que a média anual da temperatura do ar apresentou aumento de $1,35^{\circ} \mathrm{C}$ no período de 40 anos. Esse fato corrobora com as projeções apresentadas pelo IPCC, cujo aumento da temperatura média global é $0,15^{\circ} \mathrm{C}$ a $0,30^{\circ} \mathrm{C}$ por década.

\section{Elaboração dos arquivos climáticos futuros}

O levantamento das pesquisas publicadas em plataformas científicas sobre estudos de projeções climáticas futuras possibilitou o conhecimento da metodologia indicada pelo IPCC para a elaboração dos arquivos climáticos com influência do aquecimento global (SONG; YE, 2017; SABUNAS; KANAPICKAS, 2017; WANG; LIU; BROWN, 2017; INVIDIATA; GHISI, 2016; TRIANA, LAMBERTS; SASSI, 2016; CASAGRANDE; ALVAREZ, 2013). Por meio destes estudos, identificou-se a metodologia “morphing”, publicada por Belcher, Haker e Powell (2005), para a elaboração dos arquivos climáticos futuros. Essa metodologia modifica um conjunto de variáveis climáticas históricas (1961-1990) de 8.760 horas anuais, sem a influência da urbanização, e incorpora os efeitos do aquecimento global nos arquivos climáticos, obtendo, assim, as projeções de dados climáticos futuros.

A percepção do processo empregado por este método evidenciou a complexidade de elaborar cenários climáticos futuros com a visão de aplicação em softwares de desempenho termoenergético de habitações. No sentido de facilitar e consolidar as operações algorítmicas citadas, o grupo de pesquisa "Sustainable Energy Research Group" (SERG), da Universidade de Southampton, no Reino Unido, desenvolveu junto com a Microsoft ${ }^{\circledR}$ a ferramenta "Climate Change World Weather Generator" (CCWorldWeatherGen), incorporada ao Software Excel, disponibilizada gratuitamente. A ferramenta consiste de uma planilha eletrônica que integra os arquivos de extensão EnergyPlus Weather Data (EPW) ao Modelo Climático Global (MCG) "Hadley Centre Coupled Model version 3" (HadCM3) que, por sua vez, consiste de um modelo acoplado oceano-atmosfera, com resolução de $417 \mathrm{~km} \times 278 \mathrm{~km}$ na região do Equador e de $295 \mathrm{~km}$ x $278 \mathrm{~km}$ aos $45^{\circ}$ de Latitude, compondo-se do cenário de emissões do cenário A2 do Quarto Relatório (AR4) do IPCC, para os time-slice 2020 (período de 2011-2040) e 2050 (período de 2041-2070). Desta forma, utilizou para a criação dos arquivos climáticos futuros o arquivo climático atual (EPW), obtido do Solar and Wind Energy Resource Assessment (SWERA) (DOE, 2011).

Para maiores detalhes da aplicação da metodologia, podem ser consultados Guarda et al. (2019); Guarda, Durante e Callejas (2018), Song e Ye (2017), Sabunas e Kanapickas 
guARDA, E. L. A. da; DURANTE, L. C.; CALlejAS, I. J. A.

Impacto das mudanças climáticas no ambiente térmico interno de habitação unifamiliar em Cuiabá-MT

(2017), Wang, Liu e Brown (2017), Invidiata e Ghisi (2016), Triana, Lamberts e Sassi (2016), bem como Casagrande e Alvarez (2013).

\section{Definição do objeto de estudo}

Tomou-se como objeto de estudo uma habitação residencial unifamiliar padrão (HISp), localizada na cidade de Cuiabá-MT, com $39,18 \mathrm{~m}^{2}$ de área construída, contendo Sala/Cozinha (17,44m²), Quarto $1\left(7,78 \mathrm{~m}^{2}\right)$, Quarto $2\left(7,57 \mathrm{~m}^{2}\right)$ e Banheiro $\left(1,75 \mathrm{~m}^{2}\right)$ (Figura 1). A cobertura possui duas águas com beirais de $30 \mathrm{~cm}$. O pé-direito é de $3,00 \mathrm{~m}$.
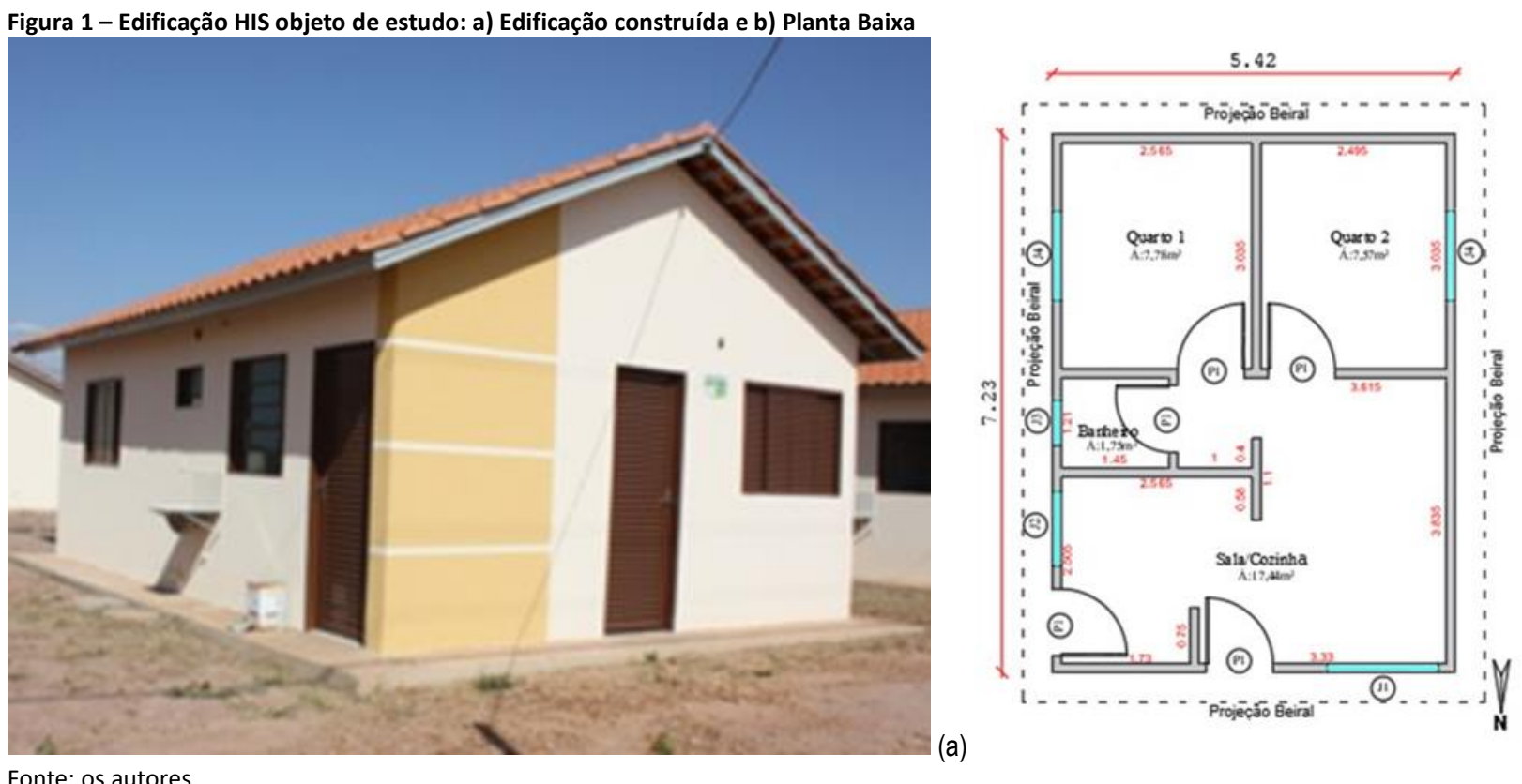

(a)

As esquadrias dos quartos e sala são metálicas, do tipo veneziana e vidro, de correr, com quatro folhas (duas fixas e duas móveis), e da cozinha, do tipo basculantes. As portas externas são de chapas metálicas e as internas de madeira.

A área mínima de abertura para ventilação dos ambientes de permanência prolongada em relação à área útil de piso é de 10 a 15\% (ABNT, 2005), maior ou igual a 7\% (ABNT, 2013) e maior ou igual a $5 \%$ (INMETRO, 2012). Como esses percentuais variam de acordo com as normativas, as dimensões das esquadrias foram ajustadas com a finalidade de atender aos pré-requisitos do RTQ-R (INMETRO, 2012), que é a menos restritiva dentre as três, mantendo-se as tipologias, conforme Tabela 2.

\begin{tabular}{|c|c|c|c|c|c|}
\hline \multirow[b]{2}{*}{ Edificação } & Ambientes: & \multicolumn{2}{|c|}{ Sala/Cozinha } & Quarto 1 & Quarto 2 \\
\hline & Área de piso $\left(\mathrm{m}^{2}\right)$ & \multicolumn{2}{|c|}{17,22} & 7,78 & 7,57 \\
\hline \multirow{6}{*}{ Original } & Dimensões (m) & $1,50 \times 1,00$ & $1,00 \times 1,00$ & $1,20 \times 1,00$ & $1,20 \times 1,00$ \\
\hline & Área $\left(\mathrm{m}^{2}\right)$ & 1,5 & 1 & 1,2 & 1,2 \\
\hline & Fator ventilação (\%) & 40 & 70 & 40 & 40 \\
\hline & Fator iluminação (\%) & 45 & 80 & 45 & 45 \\
\hline & Área efetiva de ventilação $\left(\mathrm{m}^{2}\right)$ & 0,6 & 0,7 & 0,48 & 0,48 \\
\hline & Área efetiva de iluminação $\left(\mathrm{m}^{2}\right)$ & 0,68 & 0,65 & 0,54 & 0,54 \\
\hline \multirow{6}{*}{$\begin{array}{c}\text { Ajustada } \\
\text { para } \\
\text { atender à } \\
\text { NBR } 15575 \\
\text { (ABNT, } \\
\text { 2013) }\end{array}$} & Dimensões (m) & $1,50 \times 1,10$ & $1,20 \times 1,10$ & $1,20 \times 1,10$ & $1,20 \times 1,10$ \\
\hline & Área $\left(\mathrm{m}^{2}\right)$ & 1,65 & 1,32 & 1,32 & 1,32 \\
\hline & Fator ventilação (\%) & 40 & 70 & 40 & 40 \\
\hline & Fator iluminação (\%) & 45 & 80 & 45 & 45 \\
\hline & Área efetiva de ventilação $\left(\mathrm{m}^{2}\right)$ & 0,66 & 0,92 & 0,52 & 0,52 \\
\hline & Área efetiva de iluminação $\left(\mathrm{m}^{2}\right)$ & 0,74 & 1,05 & 0,59 & 0,59 \\
\hline
\end{tabular}

Fonte: os autores. 
Os sistemas construtivos e materiais da envoltória da HISp são os tradicionalmente adotados em habitações de interesse social em todas as regiões do Brasil (BRASIL, 2007). Tal como caracterizado por Guarda et al. (2018), é incipiente a preocupação com essa especificação e sua adequação às condições climáticas dos locais de implantação, resultando em edifícios com baixo desempenho térmico e energético, o que compromete as condições de conforto térmico interno e aumenta o consumo de energia para refrigeração (GUARDA et al., 2019; TRIANA; LAMBERTS; SASSI, 2015; INVIDIATA; GHISI, 2018). Com isso, justifica-se a adoção do objeto de estudo.

Após o ajuste das aberturas mínimas, procedeu-se à simulação computacional preliminar da HISp, verificando-se a classificação de eficiência energética de sua envoltória em " $D$ ", segundo os procedimentos do RTQ-R, detalhados no item a seguir. Para alcançar a classificação de eficiência "A" e o valor mínimo de Graus-Hora de Resfriamento (GHR) de $12.566^{\circ} \mathrm{Ch}$ estabelecido no RTQ-R (INMETRO, 2012), foram feitas alterações construtivas na envoltória da HISp, obtendo-se a habitação doravante denominada HISa (Tabela 3). As escolhas são justificadas pela priorização de soluções que permitissem a implementação nas habitações já construídas, que utilizassem materiais com disponibilidade na região e de custo acessível para a população de baixa renda.

\begin{tabular}{|c|c|c|c|c|c|c|c|c|}
\hline Habitação & Envoltória & & Composição & $\begin{array}{l}\text { Espessura } \\
(\mathrm{cm})\end{array}$ & $\begin{array}{l}\text { Absortância } \\
\text { solar }\end{array}$ & $\begin{array}{c}\mathrm{Rt}^{*} \\
\left(\mathrm{~m}^{2} \mathrm{~K} / \mathrm{W}\right)\end{array}$ & $\begin{array}{c}U \\
\left(W / m^{2} K\right)\end{array}$ & $\begin{array}{c}\mathrm{C} \\
\left(\mathrm{J} / \mathrm{m}^{2} \mathrm{~K}\right)\end{array}$ \\
\hline \multirow{5}{*}{ HISp } & & \multirow{3}{*}{$\begin{array}{l}\text { Parede } \\
\text { externa e } \\
\text { interna }\end{array}$} & Argamassa & 2,50 & 0,30 & \multirow{3}{*}{0,2991} & \multirow{3}{*}{3,34} & \multirow{3}{*}{16687} \\
\hline & & & $\begin{array}{c}\text { Tijolo } \\
\text { Cerâmico }\end{array}$ & 9,00 & 0,85 & & & \\
\hline & & & Argamassa & 2,50 & 0,30 & & & \\
\hline & & \multirow{2}{*}{ Cobertura } & $\begin{array}{c}\text { Telha } \\
\text { Cerâmica }\end{array}$ & 1,00 & 0,85 & \multirow{2}{*}{0,4795} & \multirow{2}{*}{2,08} & \multirow{2}{*}{41,92} \\
\hline & & & Forro de PVC & 1,00 & 0,20 & & & \\
\hline \multirow{9}{*}{ HISa } & & \multirow{4}{*}{$\begin{array}{l}\text { Parede } \\
\text { Externa }\end{array}$} & Argamassa & 2,50 & 0,20 & 1,298 & 0,770 & 169,38 \\
\hline & & & EPS & 4,00 & 0,20 & & & \\
\hline & & & $\begin{array}{c}\text { Tijolo } \\
\text { Cerâmico }\end{array}$ & 9,00 & 0,85 & & & \\
\hline & & & Argamassa & 2,50 & 0,20 & & & \\
\hline & & \multirow{3}{*}{$\begin{array}{l}\text { Parede } \\
\text { Interna }\end{array}$} & Argamassa & 2,50 & 0,20 & 0,299 & 3,344 & 164,61 \\
\hline & & & Tijolo & 9,00 & 0,85 & & & \\
\hline & & & Argamassa & 2,50 & 0,20 & & & \\
\hline & & \multirow{2}{*}{ Cobertura } & $\begin{array}{c}\text { Telha } \\
\text { Cerâmica }\end{array}$ & 2,00 & 0,20 & 1,057 & 0,946 & 41,92 \\
\hline & & & PVC & 1,00 & 0,20 & & & \\
\hline
\end{tabular}

*Resistência Superficial Externa = 0,04, Resistência Superficial Interna fluxo horizontal = 0,13 e Resistência Superficial Interna fluxo ascendente = 0,17. . Fonte: Relatório HTML do EnergyPlus.

\section{Simulação computacional}

A simulação computacional permite analisar o desempenho termoenergético de uma habitação, considerando a tipologia, geometria, sistemas construtivos, clima de implantação e ocupação de seu interior. Utilizou-se o software EnergyPlus do Departamento de Energia dos Estados Unidos (DOE), validado pela Standard 140-2004 (ASHRAE, 2004), pela possiblidade de inserção dos arquivos climáticos (epw) atual e futuros nas simulações. 
Foram consideradas duas pessoas por dormitório e quatro pessoas na sala, com taxa metabólica de $60 \mathrm{~W} / \mathrm{m}^{2}$ (sentado ou assistindo TV) e $45 \mathrm{~W} / \mathrm{m}^{2}$ (dormindo ou descansando), respectivamente, conforme Manual RTQ-R 4.2, versão 1.0 (INMETRO, 2012). Os padrões de iluminação foram configurados para os ambientes de permanência prolongada, considerando dias de semana e finais de semana (Tabela 4). A densidade de potência instalada da iluminação recomendada é de $5,0 \mathrm{~W} / \mathrm{m}^{2}$ para dormitórios e de $6,0 \mathrm{~W} / \mathrm{m}^{2}$ para sala (INMETRO, 2012). As cargas internas de equipamentos devem ser modeladas somente para a sala, com o período de 24 horas e a potência de $1,5 \mathrm{~W} / \mathrm{m}^{2}$ (INMETRO, 2012).

Tabela 4. Rotinas de ocupação e iluminação para dias de semana e finais de semana conforme RTQ-R.

\begin{tabular}{ccccc}
\hline \multirow{2}{*}{ Rotinas } & \multicolumn{2}{c}{ Dormitórios } & \multicolumn{2}{c}{ Sala/Cozinha } \\
\cline { 2 - 5 } & Dia de Semana & Finais de Semana & Dia de Semana & Finais de Semana \\
\hline Ocupação & 21h às $8 \mathrm{~h}$ & 21h às $10 \mathrm{~h}$ & $14 \mathrm{~h}$ às $21 \mathrm{~h}$ & $11 \mathrm{~h}$ às $21 \mathrm{~h}$ \\
\hline Iluminação & 21h às 22h & 21h às 22h & 17h às 21h & 11h às 12h e das 17h às 21h \\
\hline Fonte: Adaptado de RTQ-R (INMENTRO, 2012).
\end{tabular}

As simulações de edifícios condicionados artificialmente devem ser modeladas utilizando de sistema de condicionamento de ar nos ambientes de permanência prolongada (sala e dormitório). Para análise do consumo energético, considerou-se um sistema de condicionamento artificial, modelo split, com COP para resfriamento de 3,oW/W (nível "A" na rotulagem energética brasileira) e taxa de fluxo de ar por pessoa de $0,00944 \mathrm{~m}^{3}$, modo de operação contínua do ventilador (eficiência do ventilador 0,7 e eficiência do motor 0,9 ), com período de funcionamento das $21 \mathrm{~h}$ às $8 \mathrm{~h}$, mantendo temperatura de $24^{\circ} \mathrm{C}$ para refrigeração. No período das $9 \mathrm{~h}$ às $20 \mathrm{~h}$, considerou-se a habitação Naturalmente Ventilada (NV) (INMETRO, 2012).

Considerando que o consumo de energia elétrica do sistema de iluminação e de equipamentos permaneceu constante em todas as simulações, visto que as rotinas não foram alteradas para os cenários, subtraiu-se o consumo desses sistemas e, verificou-se a variação de consumo de energia elétrica devida ao sistema de condicionamento de ar artificial (HVAC). Destaca-se que os valores de consumo tratam de consumo relativo, estabelecido com base nos padrões de uso e operação do RTQ-R, não sendo, portanto, consumo real.

\section{Resultados}

\section{Variáveis climatológicas}

Em Cuiabá, os resultados das simulações evidenciaram que os efeitos climatológicos futuros evidenciam a possibilidade do aumento das médias mensais de temperatura do ar externo e radiação solar e diminuição da umidade relativa do ar.

O aumento das temperaturas médias de bulbo seco anual foi estimado em $1,51^{\circ} \mathrm{C}$ no cenário $2020 ; 3,17^{\circ} \mathrm{C}$ no cenário $2050 ;$ e $5,75^{\circ} \mathrm{C}$ no cenário 2080 , alcançando, neste último, valores superiores a $31^{\circ} \mathrm{C}$. A média anual no Cenário Base é de $26,73^{\circ} \mathrm{C}$, passando para $28,24^{\circ} \mathrm{C}, 29,90^{\circ} \mathrm{C}$ e $32,48^{\circ} \mathrm{C}$, nos cenários 2020 (2011-2040), 2050 (2041-2070) e 2080 (20712100), respectivamente. Verificou-se que o aumento da temperatura de bulbo seco é mais expressivo entre os cenários de 2050 e 2080.

A umidade relativa do ar média anual sofreu redução de $4,33 \%$ no cenário $2020,8,67 \%$ no cenário 2050 e $15,40 \%$ no cenário 2080, atingindo, neste último, valores inferiores a $67 \%$. A média anual no Cenário Base é de $69,08 \%$, passando para $64,75 \%$, 60,41\% e 53,67\%, em 2020, 2050 e 2080, respectivamente. Ressalta-se que essa redução está relacionada diretamente com o aumento da temperatura média do ar (Figura 2). 
GUARDA, E. L. A. da; DURANTE, L. C.; CALLEJAS, I. J. A.

Impacto das mudanças climáticas no ambiente térmico interno de habitação unifamiliar em Cuiabá-MT

Esses resultados corroboram com Triana, Lamberts e Sassi (2018), Guarda, Durante e Callejas (2018), Alves (2014) e Casagrande (2013).

Figura 2 - a) Temperatura de Bulbo Seco $\left({ }^{\circ} \mathrm{C}\right)$ e b) Umidade Relativa do ar no Cenário Base (1961-1990) e nos Cenários Futuros de 2020, 2050 e 2080

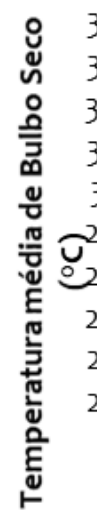

37
35
34
32
31
$\widetilde{\sigma}_{29}$
28
26
25
23

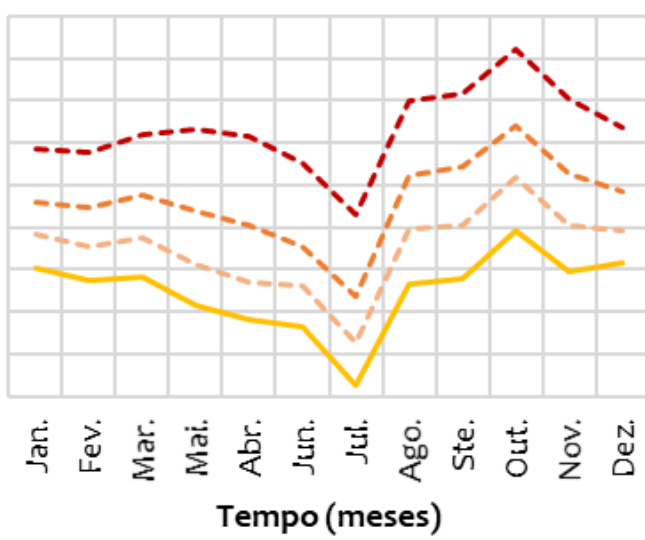

Tempo (meses)

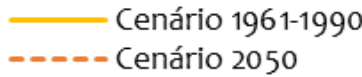

- - Cenário 2020

(a)

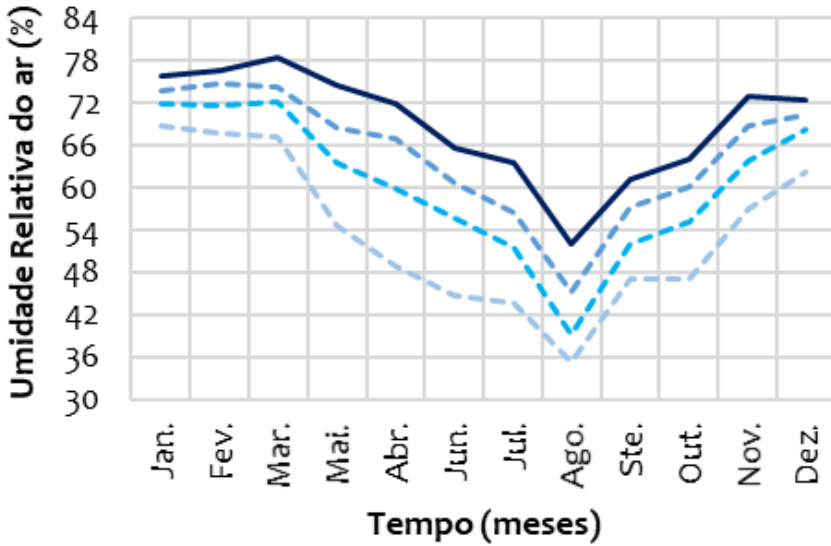

Cenário 1961-1990

- - - Cenário 2020

----- Cenário 2080

(b)

Fontes: os autores.

\section{Eficiência energética conforme RTQ-R (INMETRO, 2012)}

Analisando-se as previsões apresentadas nos quatro cenários climáticos pesquisados, verificou-se que ambas as habitações apresentaram aumento progressivo de GrausHora de Resfriamento (GHR), sendo que as maiores diferenças e as piores classificações de eficiência energética ocorreram nos cenários 2050 e 2080, respectivamente (Figura 3).

Figura 3 - Classificação da eficiência energética da envoltória da HISp e HISa nos Cenários Base (1961-1990) e Futuros (2020, 2050 e 2080)

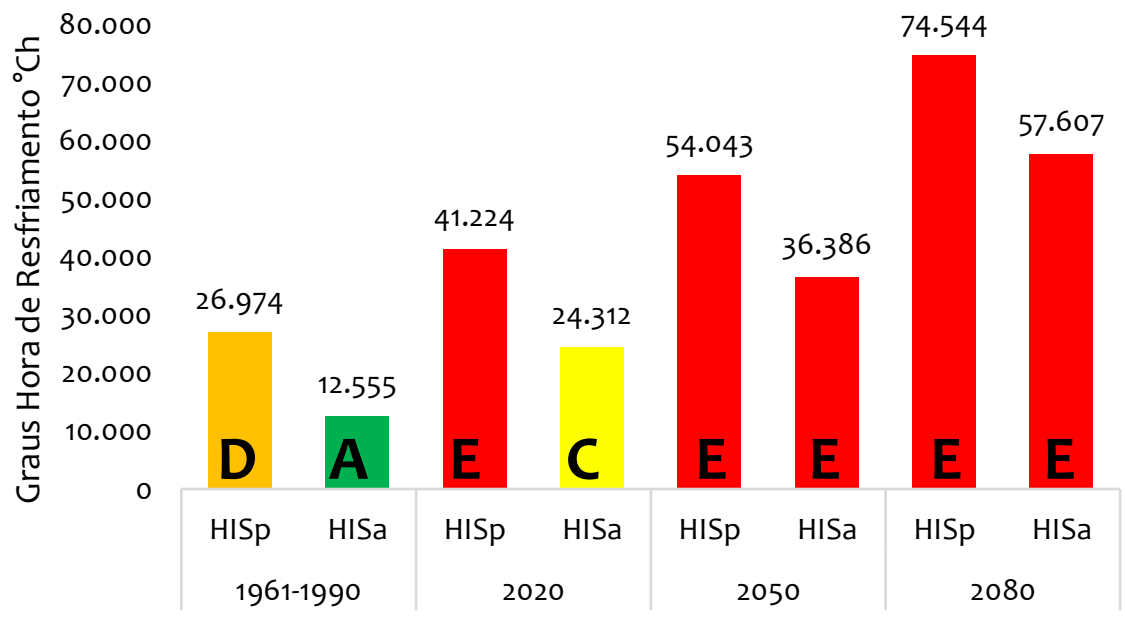

Fonte: os autores.

O valor de GHR da HISp aumentou em $63,81 \%\left(+47.570^{\circ} \mathrm{Ch}\right)$ até o cenário de 2080 , em relação ao cenário atual (1961-1990), enquanto que na HISa, esse aumento foi de $78,20 \%$ $\left(+45.052^{\circ} \mathrm{Ch}\right)$. As classificações de eficiência energética são " $D$ " e "A" no cenário atual, passando para "E" nos cenários de 2050 e 2080, respectivamente. Verificou-se que as intervenções construtivas adotadas na HISa, mitigaram os efeitos das mudanças climáticas até o cenário de 2020, para a qual obteve classificação de eficiência energética "C". Esses aumentos estão relacionados às condições climáticas externas, que 
consequentemente, refletem nas condições internas das habitações. Assim, observa-se que a adaptação da habitação atual por meio de intervenções construtivas, alcançou melhorias na eficiência energética, porém são insuficientes para manutenção do padrão "A", sinalizando um elevado consumo para fins de resfriamento.

\section{Consumo relativo energético}

Os meses de setembro a dezembro apresentaram maiores picos de consumo relativo de energia elétrica para condicionamento térmico artificial (HVAC). Na HISp, para o Cenário Base, o mês de outubro apresentou maior consumo relativo, com 398kWh e o mês de julho, o menor consumo, de $190 \mathrm{kWh}$. O mês de outubro apresentou aumento de 90,58\% até o cenário de 2080 , em relação ao Cenário Base. Os valores foram de $+35 \%$ (526kWh) em 2020, de $+57 \%$ (664kWh) em 2050 e de 90\% (815kWh) em 2080. Em julho, o aumento foi de $16 \%$ em 2020, $49 \%$ em 2050 e $120 \%$ em 2080, passando para $227 \mathrm{kWh}$, $295 \mathrm{kWh}$ e $419 \mathrm{kWh}$, respectivamente (Figura 4a).

O desempenho da HISa no Cenário Base foi semelhante ao da HISp. Os meses de outubro e julho apresentaram maior e menor consumo relativo, com valores de $271 \mathrm{kWh}$ $144 \mathrm{kWh}$, respectivamente. $\mathrm{O}$ aumento do consumo relativo para resfriamento no mês de outubro foi de 139\% no cenário de 2080, em relação ao Cenário Base. Em 2020 e 2050, o aumento foi progressivo, porém maior que na HISp, apresentando aumento de $57 \%$ e 91\%, respectivamente, passando para 401, 479 e 590kWh, em 2020, 2050 e 2080, respectivamente. $O$ mês de julho também sofreu elevação, porém, menor que o mês de outubro. A HISa apresentou elevação de consumo de 10\% em 2020, de 46\% em 2050 e de $139 \%$ em 2080, passando para 130kWh, 173kWh e 266kWh, respectivamente (Figura 4b).

Figura 4. Comportamento do consumo relativo para resfriamento das habitações Cenários Base (1961-1990) e Futuros (2020, 2050 e 2080): a) Consumo relativo da HISp e b) Consumo relativo da HISa

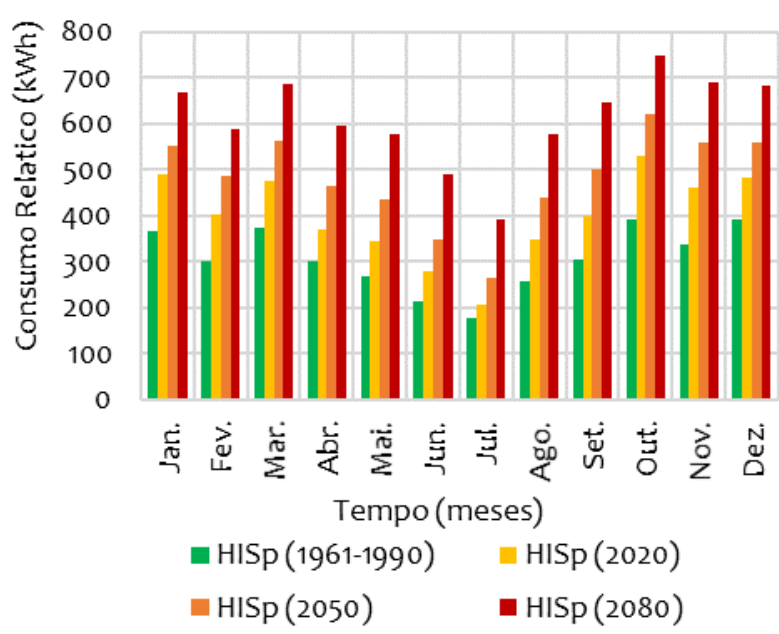

(a)

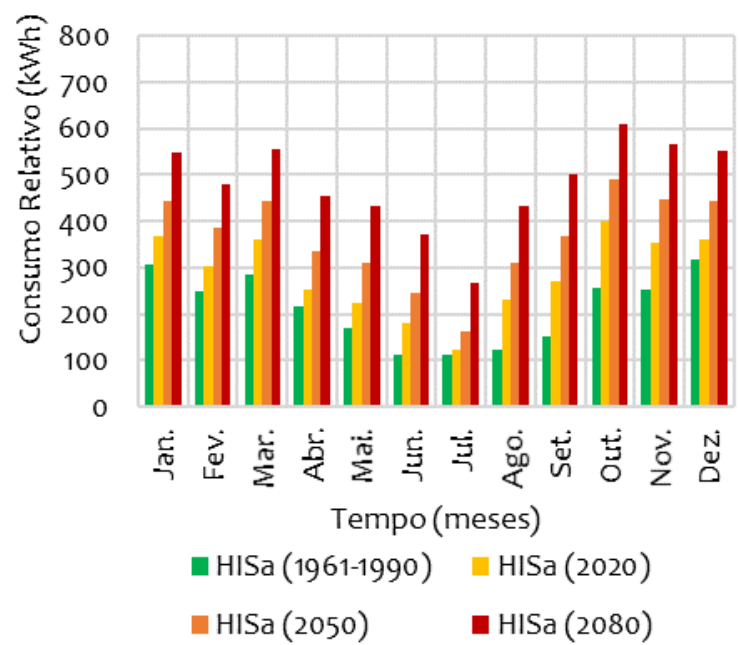

(b)

Fonte: os autores.

O consumo relativo anual (iluminação, equipamentos e ar condicionado, somados) da HISp e HISa sofreu aumento de $+4.048 \mathrm{kWh}$ e de $+2.993 \mathrm{kWh}$ até o final do século, comparando com o Cenário Base. $\mathrm{O}$ aumento do consumo relativo é progressivo, tanto na HISp quanto na HISa. Na HISp tem os aumentos de: 27,12\% em 2020, 51.29\% em 2050, e $89,46 \%$ em 2080 , todos em relação ao cenário atual. Na HISa, os aumentos foram de 26,08\% em 2020, 51.44\% em 2050 e 90,67\% em 2080, todos em relação ao cenário atual (Figura 5-a).

No cenário atual, a HISp e a HISa apresentam consumo relativo total de $115,49 \mathrm{kWh} / \mathrm{m}^{2} \mathrm{e}$ $84,25 \mathrm{kWh} / \mathrm{m}^{2}$, respectivamente. Em 2080, os respectivos consumos projetados são de 
$218,81 \mathrm{kWh} / \mathrm{m}^{2}$ e $160,64 \mathrm{kWh} / \mathrm{m}^{2}$, o que representa um aumento de $90 \%$, em ambos os casos (Figura 5-b).

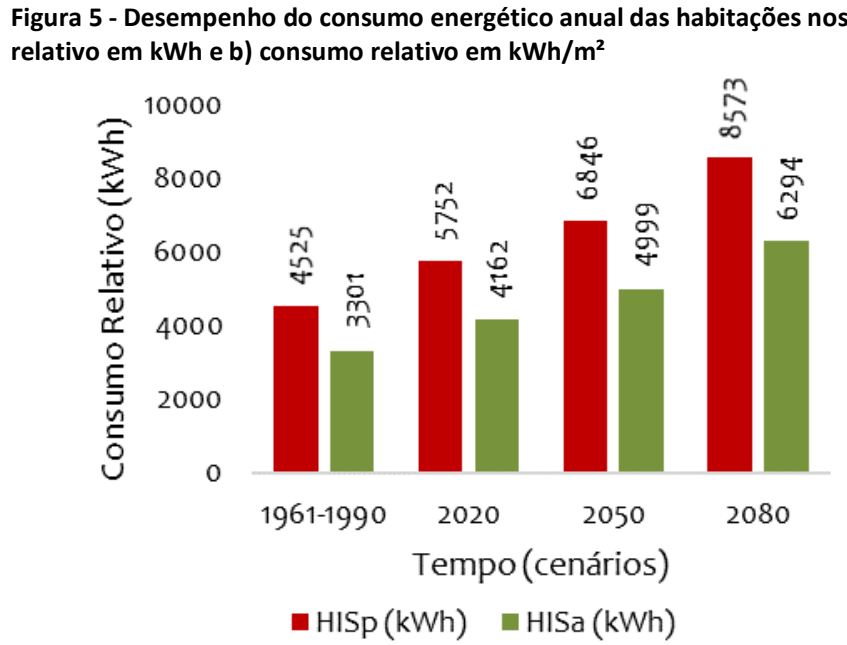

(a)

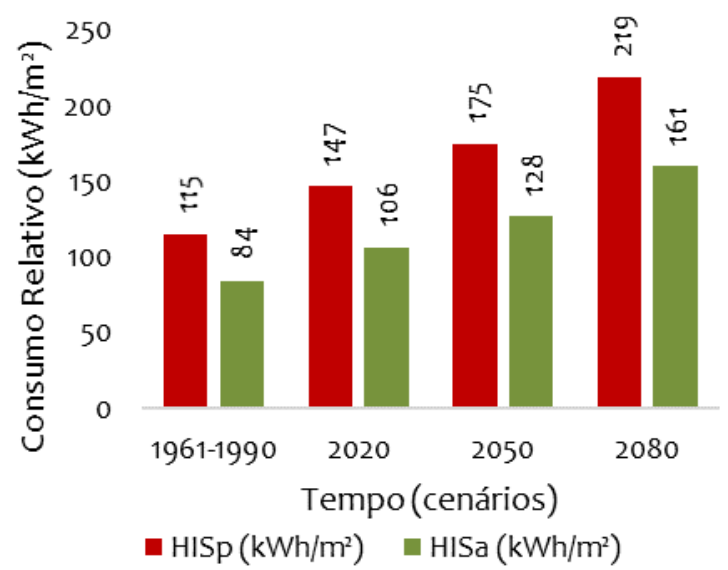

(b)

Fonte: os autores.

Destaca-se que o uso de HVAC influenciou diretamente no aumento do consumo energético da HISp, sendo de $3.693 \mathrm{kWh}$ no cenário atual, de $4.802 \mathrm{kWh}$ em 2020 , de $5.797 \mathrm{kWh}$ em 2050 e de $7.347 \mathrm{kWh}$ em 2080. Na HISa, os valores foram de $2.554 \mathrm{kWh}$, $3.430 \mathrm{kWh}, 4.389 \mathrm{kWh}$ e $5.766 \mathrm{kWh}$, nos Cenário Base, 2020, 2050 e 2080. O sistema de iluminação e equipamentos representou $670 \mathrm{kWh}$ do consumo relativo em cada cenário analisado.

Alguns autores apresentam resultados com comportamentos semelhantes, com aumento da demanda energética de resfriamento e redução de aquecimento, como Wang, Liu e Brown (2017), que investigaram cinco cidades dos Estados Unidos e encontraram maiores aumentos no consumo total de energia dos edifícios em Miami $(+12,8 \%)$, Phoenix $(+14,0 \%)$ e Los Angeles $(+10,7 \%)$ do que em Washington DC $(+4,0 \%)$ e Akron (+3,7\%). No Brasil, Casagrande e Alvarez (2013) observaram um aumento médio no consumo anual de 10,7\%, 17,3\% e 26,5\%, para os períodos de 2020, 2050 e 2080 , respectivamente, em relação ao consumo atual, para a cidade de Vitória-ES. Invidiata e Ghisi (2016) analisaram uma Habitação de Interesse Social para Curitiba, Florianópolis e Belém e obtiveram que a demanda de energia de aquecimento e resfriamento é diferente entre as cidades, variando de $20 \mathrm{kWh} / \mathrm{m}^{2}$, em Curitiba, a $102 \mathrm{kWh} / \mathrm{m}^{2}$, em Belém. Porém, os resultados mostram que há um aumento da demanda de energia ao longo dos anos em comparação com a demanda atual.

Os resultados demostram que os efeitos do aquecimento global modificaram o consumo energético para resfriamento em ambas as habitações. No entanto, a HISa apresentou menor consumo no cenário atual e nas projeções climáticas futuras, demostrando sua resiliência ao aquecimento global e comprovando a necessidade de que sejam implementadas, nos dias atuais, estratégias e diretrizes de projetos para garantirem a habitabilidade no futuro.

\section{Conclusão}

Evidenciou-se que os efeitos previsionais das mudanças climáticas, segundo a metodologia adotada, irão impactar as variáveis climatológicas do local de estudo Cuiabá/MT, localizada na cidade de Cuiabá-MT, região Centro-Oeste do Brasil, 
aumentando gradualmente a temperatura do ar e a radiação solar, e reduzindo a umidade relativa do ar.

O estudo parte de uma habitação padrão, cujo projeto (materiais construtivos, dimensões e volumetria) é recorrente em todo o território nacional (HISp), cuja classificação de eficiência energética da envoltória é " $D$ ". Seguindo as diretrizes atuais da normativa bioclimática e da regulamentação de eficiência energética brasileira, alterou-se a especificação de sua envoltória para que fosse alcançada classificação de eficiência energética " $A$ " (HISa), incorporando medidas combinadas de aumento da resistência térmica.

A partir disso, a eficiência energética da envoltória e o consumo relativo de energia foram analisados para os cenários das emissões A2 do Quarto Relatório do IPCC (AR4) e comparados ao atual, que corresponde ao período de 1961-1990, considerado como aquele sem os efeitos das alterações decorrentes do fenômeno de mudanças climáticas.

Verificou-se que a eficiência da envoltória de ambas (HISp e HISa) sofrerá com os possíveis efeitos das mudanças climáticas, sendo gradualmente reduzida nos cenários futuros. Até o cenário de 2080, o valor dos Graus-Hora de Resfriamento (GHR) da HISp aumentou em $176 \%\left(47.570^{\circ} \mathrm{Ch}\right)$. Já na HISa, o aumento foi menor $\left(45.052^{\circ} \mathrm{Ch}\right)$, evidenciando sua maior adaptabilidade em relação à HISp.

As classificações de eficiência energética obtidas para HISp e HISa são "D" e " $A$ " no cenário atual, respectivamente, ambas passando para " $\mathrm{E}$ " nos cenários de 2050 e 2080. Em relação ao cenário atual, HISp e HISa apresentaram aumento de 89,46\% e 90,67\% na demanda energética no cenário de 2080 , respectivamente.

Constata-se que a habitação padrão já apresenta baixo desempenho termoenergético no cenário atual. No entanto, com os efeitos previsionados de aquecimento global, as condições de desempenho térmico e, consequentemente, a habitabilidade da edificação, poderão se tornar ainda mais comprometidas. No cenário de mais longo prazo (2080), esses efeitos também se estendem à HISa.

Os resultados deste estudo ressaltam a importância de adequação das normatizações e regulamentos brasileiros quanto aos padrões estabelecidos dos níveis de desempenho termoenergético, bem como de atualizações regulares, para assegurar a incorporação de medidas que garantam eficiência energética não só para hoje, mas para que se mantenham diante dos possíveis efeitos das mudanças climáticas projetados.

Por fim, os modelos climáticos previsionais geram condições que podem ou não prevalecer nos períodos futuros, devendo os valores absolutos apresentados nessa pesquisa serem tratados como uma possível tendência. Contudo, os erros potenciais nos dados climáticos futuros gerados não invalidam a ferramenta previsional adotada, e podem ser usados na antecipação do comportamento do ambiente construído nos cenários de mudanças climáticas.

\section{Agradecimentos}

À Fundação de Amparo à Pesquisa do Estado de Mato Grosso (FAPEMAT) pela bolsa de mestrado concedida no período de elaboração deste artigo, sob número de edital 017/2015. Ao Laboratório de Tecnologia e Conforto Ambiental (LATECA) da Universidade Federal de Mato Grosso. 


\section{Referências}

ABNT - ASSOCIAÇÃO BRASILEIRA DE NORMAS TÉCNICAS. NBR 15.220-2: Desempenho térmico de edificações Método de cálculo da transmitância térmica, da capacidade térmica, do atraso térmico e do fator solar de elementos e componentes de edificações. Rio de Janeiro, 2005.

ABNT - ASSOCIAÇÃO BRASILEIRA DE NORMAS TÉCNICAS. NBR 15.575-1: Edificações habitacionais - Desempenho Requisitos gerais. Rio de Janeiro, 2013.

ALVES, C. A. Resiliência das Edificações às mudanças climáticas na região metropolitana de São Paulo - Estudo de caso: Desempenho térmico de edifícios residenciais para idosos. 2014. Dissertação (Mestrado) - Faculdade de Arquitetura e Urbanismo, Universidade de São Paulo. São Paulo, 2014. DOI: https://doi.org/10.11606/D.16.2015.tde28082015-101646

BELCHER, S; HACKER, J; POWELL, D. Constructing design weather data for future climates. Building Services Engineering Research and Technology, v.26, p. 46-61, 2005. https://doi.org/10.1191/0143624405bt1120a

BRASIL. Caixa Econômica Federal. Standard Project Popular Houses 42m. 2007. Disponível em: http://www.caixa.gov.br/site/paginas/downloads.aspx/ Acesso em: 8 Abri. 2018.

CALLEJAS, I. J. A.; BIUDES. M. S.; MACHADO, N. G.; DURANTE, L. C.; LOBO, F. A. Patterns of Energy Exchange For Tropical Urban And Rural Ecosystems Located In Brazil Central. Journal of Urban and Environmental Engineering (JUEE), v.13, n.1, p.69-79, 2019. https://doi.org/10.409o/juee.2019.v13n1.069079

CAMPELO JÚNIOR, J. H; PRIANTE FILHO, N; CASEIRO, F. T. Caracterização macroclimática de Cuiabá. In: ENCONTRO NACIONAL DE ESTUDOS SOBRE O MEIO AMBIENTE, 1991, Londrina. Anais [...]. Londrina: Universidade Estadual de Londrina, Núcleo de Estudos do Meio Ambiente, 1991, 773p.

CASAGRANDE, B. ALVAREZ, C. Preparação de arquivos climáticos futuros para avaliação dos impactos das mudanças climáticas no desempenho termoenergético de edificações. Ambiente Construído, Porto Alegre, v.13, n.4, p. 173-187, 2013. DOI: https://doi.org/10.1590/S1678-86212013000400012

DE WILDE P; COLEY D. The implications of a changing climate for buildings. Build Environment, v.55, n.1-7, 2012.

DOE - DEPARMENT OF ENERGY. EnergyPlus. Energyplus.net. Disponível em: https://energyplus.net. Acesso em: 16 de maio de 2018 .

DU, H; EDGE, J; UNDERWOOD, C. Modelling the Impacts of New UK Future Weather Data on a School Building. Building Simulation, p. 538-545, 2011. DOI: https://doi.org/10.1016/j.buildenv.2012.03.014

DUARTE, D. H. S. Padrões de ocupação do solo e microclimas urbanos na região de clima tropical continental. 2000. f. 296. Tese (Doutorado) - Faculdade de Arquitetura e Urbanismo da Universidade de São Paulo. São Paulo, 2000.

FAHMY, M; MAHDY, M. M; NIKOLOPOULOU. Prediction of future energy consumption reduction using GRC envelope optimization for residential buildings in Egypt. Energy and Buildings, v. 70, p. 186. Egypt, 2014. DOI: https://doi.org/10.1016/J.ENBUILD.2013.11.057

GUARDA, E. L. A; DOMINGOS, R. M. A; JORGE, S. H. M; DURANTE, L. C; SANCHES, J. C. M; LEÃO, M; CALLEJAS, I. J. A. The influence of climate change on renewable energy systems designed to achive zero energy buildings in the present: A case study in the Brazilian Savannah. Sustainable Cities and Society, v. 52, p.101843. 2019. DOI: https://doi.org/10.1016/j.scs.2019.101843

GUARDA, E. L. A; DURANTE, L. C; CALLEJAS, I. J. A; JORGE, S. H. M; BRANDÃO, R. P. Estratégias construtivas para adequação da envoltória de uma Habitação de Interesse Social às Zonas Bioclimáticas Mato-Grossenses. Revista Engineering and Science (E\&S), v. 7, n.1, p.45-57. Cuiabá, 2018. DOI: https://doi.org/10.18607/ES201876138 
INMETRO - INSTITUTO NACIONAL DE METROLOGIA, QUALIDADE E TECNOLOGIA. Requisitos técnicos da qualidade para nível de eficiência energética de edifícios residenciais. Disponível em:

http://www.pbeedifica.com.br/sites/default/files/projetos/etiquetagem/residencial/downloads/Manual_RTQR_10201 4.pdf. Acesso: 10 de julho de 2018

IPCC - INTERGOVERNMENTAL PANEL ON CLIMATE CHANGE. Climate Change 2007: Synthesis Report. Contribution of Working Groups I, II and III to the Fourth Assessment Report of the Intergovernmental Panel on Climate Change. Geneva, Switzerland, 2007.

IPCC - INTERGOVERNMENTAL PANEL ON CLIMATE CHANGE. Climate Change 2012: Managing the Risks of Extreme Events and Disasters to Advance Climate Change Adaptation. A Special Report of Working Groups I and II of the Intergovernmental Panel on Climate Change, Geneva Switzerland, 2012.

INVIDIATA, A; GHISI, E. Impact of climate change on heating and cooling energy demand in houses in Brasil. Energy and Building, v. 130, p. 20-32, 2016. DOI: https://doi.org/10.1016/j.enbuild.2016.07.067

KERSHAW, T; EAMES, M; COLEY, D. Assessing the risk of climate change for buildings: A comparison between multiyear and probabilistic reference year simulations. Building and Environment, v. 46, pp. 1303-1308, 2011. DOI: https://doi.org/10.1016/j.buildenv.2010.12.018

MACIEL, A. A; FORD, B; LAMBERTS, R. Main Influences on the design philosophy and knowledge basis to bioclimatic integration into architecture design - The example of best practices. Building and Environmental, v. 42, p. 37623773. 2007. DOI: https://doi.org/10.1016/j.buildenv.2006.07.041

OLGYAY, V. Design with climate. Bioclimatic Approach to Architectural Regionalism. p. 309-310, New Jersey, 1973.

PEEL, M. C; FINLAYSON, B. L; MCMAHON T. A. Updated world map of the Köppen-Geiger climate classification. Hydrology and Earth System Sciences, v. 11, p.1633-1644, 2007. DOI: https://doi.org/10.5194/hess-11-1633-2007

ROBERT, A; KUMMERT, M. Designing Net-Zero Energy Buildings for the Future Climate, Not for the Past. Building and Environment, v. 55, p. 150-158, 2012. DOI: https://doi.org/10.1016/j.buildenv.2011.12.014

SABUNAS A, KANAPICKAS A. Estimation of climate change impact on energy consumption in a residential building in Kaunas, Lithuania, using HEED Software. Energy Procedia, vol. 128, p. 92-9, 2017.

https://doi.org/10.1016/j.egypro.2017.09.020

SERRA, G. Pesquisa em arquitetura e urbanismo guia prático para o trabalho de pesquisadores em pós-graduação. São Paulo: Edusp/ Mandarin, 2006, 256p.

SONG, X; YE C. Climate Change Adaptation Pathways for Residential Buildings in Southern China. Energy Procedia, v. 105, p. 3062-7, 2017. https://doi.org/10.1016/j.egypro.2017.03.635

TRIANA, M. A; LAMBERTS, R. SASSI, P. Desempenho de Habitações de interesse social frente às mudanças climáticas. In: ENCONTRO NACIONAL DE TECNOLOGIA DO AMBIENTE CONSTRUÍDO. 16., 2016. São Paulo. Anais [...]. Porto Alegre:ANTAC, 2016. Disponível em: http://www.infohab.org.br/entac/2016/ENTAC2016_paper_763.pdf

WANG, L; LIU, X; BROWN H. Prediction of the impacts of climate change on energy consumption for a medium-size office building with two climate models. Energy and Buildings, v. 157, p. 218-226, dec. 2017. DOI:

https://doi.org/10.1016/j.enbuild.2017.01.007

${ }^{1}$ Emeli Lalesca Aparecida da Guarda

Arquiteta e Urbanista. Mestre em Engenharia de Edificações e Ambiental pela Universidade Federal de Mato Grosso. Doutoranda em Arquitetura e Urbanismo pela Universidade Federal de Santa Catarina. Endereço postal: Rua Engenheiro Andrei Cristian Ferreira, s/n, Trindade, Florianópolis-SC, Brasil, 88040-900. 
guARDA, E. L. A. da; DURANTE, L. C.; CALleJAS, I. J. A.

Impacto das mudanças climáticas no ambiente térmico interno de habitação unifamiliar em Cuiabá-MT

${ }^{2}$ Luciane Cleonice Durante

Engenheira Civil. Doutora em Física Ambiental pela Universidade Federal de Mato Grosso. Professora Associada da Faculdade de Arquitetura, Engenharia e Tecnologia da Universidade Federal de Mato Grosso. Endereço postal: Av. Fernando Correa da Costa, n²367, Boa Esperança, Cuiabá-MT, Brasil, 78060-900.

3 Ivan Julio Apolonio Callejas

Engenheiro Civil. Doutor em Física Ambiental pela Universidade Federal de Mato Grosso. Professor Associado da Faculdade de Arquitetura, Engenharia e Tecnologia da Universidade Federal de Mato Grosso. Endereço postal: Av. Fernando Correa da Costa, n²367, Boa Esperança, Cuiabá-MT, Brasil, 78060-900. 